\title{
Metastatic Anal Cancer: The Search for Cure
}

\author{
Bernard J. Cummings \\ Department of Radiation Oncology, Princess Margaret Hospital, University of Toronto, Canada
}

During the past three decades there have been major improvements in the management of squamous cell cancer of the anal canal. The adoption of radiation therapy with concurrent chemotherapy (usually 5-fluorouracil (5-FU) plus either mitomycin or cisplatin) in place of radical surgery has enabled about two in three patients to retain anorectal function, with overall cure rates comparable to those achieved previously by surgery [1]. But during the same period there has been little evidence that the prognosis for patients with extrapelvic metastases has improved significantly from a median of about 9-12 months and a 2 year survival rate of about $10 \%$.

In this issue Tokar and her colleagues describe how aggressive multimodality treatment, including resection of extrapelvic metastases, may extend the life of, and perhaps cure, selected patients [2]. How much can we learn from a case report such as this? With the incidence of anal cancer no more than 1 per 100,000 persons, and the finding of extrapelvic metastases in less than $5 \%$ at first presentation and as sole site of failure in no more than $10-20 \%$ of those treated previously for loco-regional cancer, we are likely to remain dependent for some time on case reports and small series for indications of how the outcome may be improved for those patients who develop extrapelvic metastases. Although several collaborative groups have demonstrated that randomized trials are possible for this uncommon cancer, even in the largest of those trials only 21 of the 285 patients treated by chemoradiation died of extrapelvic metastases without pelvic failure [3].

The most common sites of extrapelvic metastases from squamous cell anal canal cancer are the liver, lungs and extrapelvic lymph nodes, although spread to peritoneum, bone and other sites may also occur. In many patients metastases are found in more than one organ, and are usually multiple within each organ system affected. However, the time course over which metastases to multiple organs are identified, and their growth rate, sometimes suggest secondary spread from the growths in the liver so that early recognition and resection of liver metastases could be advantageous.

It is well recognized that there are significant, but not yet elucidated, biological differences between extrapelvic metastases and the primary anal cancer and its pelvic regional node metastases as reflected by the response to non-surgical treatment. Doses of radiation and concurrent chemotherapy (either 5-FU and mitomycin or 5-FU and cisplatin) which eradicate permanently often quite substantial pelvic tumor masses have, for the most part, produced no more than partial responses in metastases in the liver or other extrapelvic organs. The multiplicity of liver metastases, and their distribution throughout the liver, have restricted the doses of radiation used because of the risk of hepatic toxicity, and there is as yet little experience with new techniques such as focal high dose radiation. The most effective chemotherapy for metastatic or locally recurrent anal squamous cell cancer is 5-FU and cisplatin, which produces partial response rates of up to $50 \%$ but complete response rates of only about $15 \%$ and rarely long-term control or cure [1, 4]. Many cytotoxic drugs recently introduced to clinical practice are still being evaluated for anal cancer, and the potential of agents such as EGFR inhibitors [5] remains unexplored clinically. While the often curative role of salvage surgery for pelvic recurrence, either of the primary cancer or of regional nodes, is well established [1], the place of surgery for extrapelvic metastases is less clear with only scattered case reports. This is principally because of the multiple sites of metastases, or their detection contemporaneously with pelvic failure, that marks the common natural history of anal cancer. It may be that the relatively successful experience with excision of metastases from colorectal cancer [6] cannot be repeated in anal cancer except in a few highly selected patients.

Tokar and her colleagues observed partial responses of liver metastases to chemotherapy, and sought to consolidate con-

\begin{tabular}{ll}
\hline KARGER & ( 2006 S. Karger GmbH, Freiburg \\
Fax +497614520714 & Accessible online at: \\
$\begin{array}{l}\text { E-mail Information@Karger.de } \\
\text { www.karger.com }\end{array}$ & www.karger.com/onk \\
&
\end{tabular}

Prof. Bernard J. Cummings, MB, FRCPC

Department of Radiation Oncology

Princess Margaret Hospital/University of Toronto

610 University Avenue, Toronto, ON M5G 2M9, Canada

Tel. +1 416 946-2129, Fax -4586

E-mail Bernard.Cummings@rmp.uhn.on.ca 
trol by partial hepatectomy, a pattern they were able to repeat three times at intervals of 2 years [2]. The disease pattern they observed is atypical in that they found only a few metastases which grew fairly slowly and remained confined to the liver (although with direct extension to the diaphragm at the most recent laparotomy). At the first two surgical procedures resection of the liver metastases was microscopically incomplete and the patient was given additional chemotherapy. Following the most recent resection, which was complete although with margins of $0.1-0.4 \mathrm{~cm}$, the patient was treated with local radiation to the tumor bed without concurrent chemotherapy. In retrospect, we may speculate whether localized radiation could have been given after the first liver resection, especially if the sites of surgery were marked with clips.

On the basis of the account of this unusual patient, and the ineffectiveness of currently available alternative approaches, it would seem reasonable to manage a patient with limited metastatic anal squamous cell cancer confined to a single organ such as the liver by initial multidisciplinary assessment to determine potential resectability, treatment with several courses of 5-FU and cisplatin, restaging and reassessment, and, if no extrahepatic failure is detected, resection of the metastases. Postoperative administration of additional chemotherapy, with or without radiation, could be based on the integrity and extent of tumor-free margins, although I would be inclined to recommend maximum potentially curative treatment at the first opportunity as it may well be the only opportunity.

\section{References}

1 Cummings BJ, Swallow CJ, Ajani JA: Cancer of the anal region; in DeVita VT Jr, Hellman S, Rosenberg SA (eds): Cancer: Principles and Practice of Oncology. Philadelphia, Lippincott Williams and Wilkins, ed 7, 2005, pp 1125-1138.

2 Tokar M, Bobilev D, Zalmanov S, Geffen DB, Walfisch S: Combined multimodal approach to the treatment of metastatic anal carcinoma: Report of a case and review of the literature. Onkologie 2006; 29 DOI: $10.1159 / 000089798$.

$\checkmark 3$ UKCCCR Anal Canal Cancer Trial Working Party. Epidermoid anal cancer: Results from the UKCCCR randomized trial of radiotherapy alone versus radiotherapy, 5-fluorouracil and mitomycin C. Lancet 1996;348:1049-1054.

4 Jaiyesimi IA, Pazdur R: Cisplatin and 5-fluorouracil as salvage therapy for recurrent metastatic squamous cell carcinoma of the anal canal. Am J Clin Oncol 1993;16:536-540.

5 Le LH, Chetty R, Moore MJ: Epidermal growth factor expression in anal canal carcinoma. Am J Clin Pathol 2005;124:20-23.

6 Tepper JE, O'Connell M, Hollis D, Niedzwiecki D, Cooke E, Mayer RJ: Analysis of surgical salvage after failure of primary therapy in rectal cancer: results from Intergroup Study 0114. J Clin Oncol 2003;21:3623-3628. 